\title{
On the subdifferentiability of the difference of two functions and local minimization
}

\author{
Tijani Amahroq*, Jean-Paul Penot ${ }^{\dagger}$ and Aïcha Syam*
}

\begin{abstract}
We set up a formula for the Fréchet and $\varepsilon$-Fréchet subdifferentials of the difference of two convex functions. We even extend it to the difference of two approximately starshaped functions. As a consequence of this formula, we give necessary and sufficient conditions for local optimality in nonconvex optimization. Our analysis relies on the notion of gap continuity of multivalued maps and involves concepts of independent interest such as the notions of blunt and sharp minimizers.
\end{abstract}

Mathematics Subject Classifications (2000): 26B05, 26B25, 49K27, 90C26

Keywords: approximately starshaped function, approximately convex function, blunt minimizer, d.a.s. function, d.c. function, equi-subdifferentiability, Fréchet subdifferential, $\varepsilon$ Fréchet subdifferential, gap-continuity, local minimizer, local blunt minimizer.

\section{Introduction.}

The interest in the theory of nonsmooth d.c. (difference of convex) optimization problems has much increased in the last few years; see for instance $[9,14,13,25,31,36,38,1,55]$ and references therein. Such an interest is explained by the fact that this class of functions is large: by a result of Hartman [12], it contains the class of functions of class $C^{2}$; moreover, the distance function to a nonempty subset of a Hilbert space belongs to this class ([2]). Furthermore, this class contains the class of lower- $\mathrm{C}^{2}$ functions ([48], [47], [37]).

Let $f:=g-h$ be defined on a Banach space $E$ with $g, h: E \rightarrow \mathbb{R}$ convex. Michelot [31] proved that when $E=\mathbb{R}^{n}$ and $h$ is "locally polyhedral" at $\bar{x}$, (i.e. $\partial h(x) \subset \partial h(\bar{x})$ for all $x$ in a neighborhood of $\bar{x}$ ), then $\bar{x}$ is a local minimizer of $f$ iff $\partial h(\bar{x}) \subset \partial g(\bar{x})$. It is the main aim of the present paper to relax the assumption of [31].

Using the Fenchel $\varepsilon$-subdifferential, Hiriart-Urruty [14] established that a necessary and sufficient condition for $\bar{x} \in E$ to be an $\varepsilon$-minimizer of $f$ (in the sense that $f(\bar{x}) \leq \inf f(E)+\varepsilon)$ is that

$$
\partial_{\lambda} h(\bar{x}) \subset \partial_{\lambda+\varepsilon} g(\bar{x}) \quad \text { for all } \lambda \geq 0 .
$$

\footnotetext{
*Faculté des Sciences et Techniques Guéliz B.P. 549 Marrakech, Morocco

${ }^{\dagger}$ Faculté des Sciences, Mathématiques UMR CNRS 5142 B.P. 115564013 PAU Cedex, France
} 
He also proved that $\bar{x}$ is a global minimizer of $f$ iff

$$
\partial_{\lambda} h(\bar{x}) \subset \partial_{\lambda} g(\bar{x}) \quad \text { for all } \lambda \geq 0 \text {. }
$$

Considering the functions $g(x)=|x|$ and $h(x)=\frac{1}{2} x^{2}$, he showed that this last result is no more valid for local minimizers since 0 is a local minimizer of $f:=g-h$ and $\partial_{\lambda} g(0)=[-1,1]$, $\partial_{\lambda} h(0)=[-\sqrt{2 \lambda}, \sqrt{2 \lambda}]$, so that the above inclusion is not true for all $\lambda \geq 0$. This is due to the fact that the Fenchel subdifferential is a global notion and cannot give any information about local minimizers.

Our aim in this paper is to give necessary and sufficient conditions for a point $\bar{x} \in E$ to be a local minimizer (or rather a local blunt minimizer in the sense of Definition 6.1 below) in terms of the Fréchet subdifferentials of $f$ or the Fréchet $\varepsilon$-subdifferentials of $f$. The choice of the Fréchet subdifferential and Fréchet $\varepsilon$-subdifferentials seems to be well suited to our aims; we also deal with other subdifferentials such as the limiting subdifferential. This result will be derived by proving a formula for the Fréchet subdifferential and Fréchet $\varepsilon$-subdifferential of a difference of two approximately starshaped functions or a difference of two (approximately) convex functions.

\section{Preliminaries.}

Let $f: E \rightarrow \mathbb{R}_{\infty}:=\mathbb{R} \cup\{+\infty\}$ be a proper extended-real valued function on a Banach space $E$. We denote by $B$ the closed unit ball of $E$ and by $B^{*}$ the closed unit ball of the dual $E^{*}$ of $E$. For $\varepsilon \geq 0$, the Fenchel $\varepsilon$-subdifferential of $f$ at $\bar{x} \in \operatorname{dom} f:=\{x \in E: f(x)<\infty\}$ is the subset $\partial_{\varepsilon} f(\bar{x})$ of the topological dual $E^{*}$ of $E$ defined by

$$
\partial_{\varepsilon} f(\bar{x})=\left\{x^{*} \in E^{*}: f(x) \geq f(\bar{x})+\left\langle x^{*}, x-\bar{x}\right\rangle-\varepsilon \forall x \in E\right\} .
$$

The particular case $\varepsilon=0$ corresponds to the well known Fenchel subdifferential which is denoted by $\partial f(\bar{x})$.

Recall also that the Fréchet $\varepsilon$-subdifferential of $f$ at a point $\bar{x} \in \operatorname{dom} f$ is defined by

$$
\partial_{\varepsilon}^{F} f(\bar{x})=\left\{x^{*} \in E^{*}: \liminf _{\|v\| \rightarrow 0_{+}} \frac{f(\bar{x}+v)-f(\bar{x})-\left\langle x^{*}, v\right\rangle}{\|v\|} \geq-\varepsilon\right\}
$$

and $\partial_{\varepsilon}^{F} f(\bar{x})=\varnothing$ if $\bar{x} \notin \operatorname{dom} f$. More explicitly, for $\bar{x} \in \operatorname{dom} f$ one has

$$
\bar{x}^{*} \in \partial_{\varepsilon}^{F} f(\bar{x}) \Leftrightarrow \forall \alpha>0 \exists \delta>0 \forall x \in B(\bar{x}, \delta)\left\langle\bar{x}^{*}, x-\bar{x}\right\rangle \leq f(x)-f(\bar{x})+(\alpha+\varepsilon)\|x-\bar{x}\| .
$$

When $\varepsilon=0, \partial_{\varepsilon}^{F} f(\bar{x})$ reduces to the well known Fréchet subdifferential of $f$ at $\bar{x}$ denoted by $\partial^{F} f(\bar{x})$.

The Fréchet $\varepsilon$-subdifferential of $f$ at $\bar{x}$ is clearly related to $\partial^{F} f(\bar{x})$ by

$$
\partial_{\varepsilon}^{F} f(\bar{x})=\partial^{F}(f+\varepsilon\|\cdot-\bar{x}\|)(\bar{x}) .
$$

When $f$ is a convex function on $E$, it is known that $\partial^{F} f(\bar{x})=\partial f(\bar{x})$, and then 


$$
\partial_{\varepsilon}^{F} f(\bar{x})=\partial f(\bar{x})+\varepsilon B^{*} .
$$

If $f, g: E \rightarrow \mathbb{R}_{\infty}$ are given, as an easy consequence of the definition, for all nonnegative real numbers $\alpha, \beta$, one has

$$
\partial_{\alpha}^{F} f(\bar{x})+\partial_{\beta}^{F} g(\bar{x}) \subset \partial_{\alpha+\beta}^{F}(f+g)(\bar{x})
$$

The following operation, sometimes called the star-difference, between two subsets $C$ and $D$ of $E^{*}$ will play an important role in the sequel; it is given by

$$
C \boxminus D:=\left\{x^{*} \in E^{*}: x^{*}+D \subset C\right\} .
$$

It has been used for various purposes (optimal control theory, differential games, morphogenesis, nonsmooth analysis, regularization...) in the literature since its introduction in [45]; see [3], [5], [11], [26], [30], [35] and [46] for some references.

We will use the notion of approximately convex function introduced in [32] and studied in [4], [33] and its generalization presented in [34]; we recall these notions and present some properties we need.

Definition 2.1 A function $f: E \rightarrow \mathbb{R}_{\infty}:=\mathbb{R} \cup\{\infty\}$ is said to be approximately starshaped at $\bar{x}$ if it is finite at $\bar{x}$ and if for every $\varepsilon>0$ one can find $\delta>0$ such that for every $x \in B(\bar{x}, \delta)$ and all $t \in[0,1]$ one has

$$
f((1-t) \bar{x}+t x) \leq(1-t) f(\bar{x})+t f(x)+\varepsilon t(1-t)\|x-\bar{x}\|
$$

It is said to be approximately convex at $\bar{x}$ if it is finite at $\bar{x}$ and if for every $\varepsilon>0$ one can find $\delta>0$ such that for every $x, x^{\prime} \in B(\bar{x}, \delta)$ and any $t \in[0,1]$ one has

$$
f\left((1-t) x+t x^{\prime}\right) \leq(1-t) f(x)+t f\left(x^{\prime}\right)+\varepsilon t(1-t)\left\|x-x^{\prime}\right\| .
$$

Both classes enjoy nice stability properties ([32], [4], [33]). Moreover one has the following result.

Lemma 2.1 (a) If $f$ is approximately starshaped at $\bar{x}$, then for any $\varepsilon>0$ there exists some $\delta>0$ such that for every $x \in B(\bar{x}, \delta), x^{*} \in \partial^{F} f(x)$ and $\bar{x}^{*} \in \partial^{F} f(\bar{x})$ one has

$$
\begin{aligned}
& \left\langle\bar{x}^{*}, x-\bar{x}\right\rangle \leq f(x)-f(\bar{x})+\varepsilon\|x-\bar{x}\|, \\
& \left\langle x^{*}, \bar{x}-x\right\rangle \leq f(\bar{x})-f(x)+\varepsilon\|x-\bar{x}\| .
\end{aligned}
$$

(b) If $f$ is approximately convex at $\bar{x}$, then for any $\varepsilon>0$ there exists some $\delta>0$ such that for every $x, x^{\prime} \in B(\bar{x}, \delta), x^{*} \in \partial^{F} f(x)$ one has

$$
\left\langle x^{*}, x^{\prime}-x\right\rangle \leq f\left(x^{\prime}\right)-f(x)+\varepsilon\left\|x^{\prime}-x\right\| .
$$


Proof. (a) Given $\varepsilon>0$, we choose $\delta>0$ as in Definition 2.1, so that for every $x \in B(\bar{x}, \delta)$, $\bar{x}^{*} \in \partial^{F} f(\bar{x})$ and any $t \in[0,1]$ we have, for some function $\alpha: \mathbb{R}_{+} \rightarrow \mathbb{R}_{+}$satisfying $\lim _{t \rightarrow 0_{+}} \alpha(t)=$ 0

$$
\left\langle\bar{x}^{*}, t(x-\bar{x})\right\rangle-t \alpha(t) \leq f(\bar{x}+t(x-\bar{x}))-f(\bar{x}) \leq t(f(x)-f(\bar{x}))+\varepsilon t(1-t)\|x-\bar{x}\| .
$$

Dividing by $t$ and passing to the limit as $t \rightarrow 0_{+}$, we obtain, for every $\bar{x}^{*} \in \partial^{F} f(\bar{x})$

$$
\left\langle\bar{x}^{*}, x-\bar{x}\right\rangle \leq f(x)-f(\bar{x})+\varepsilon\|x-\bar{x}\| .
$$

Setting $s=1-t$, from the inequality

$$
f(x+s(\bar{x}-x))-f(x) \leq s(f(\bar{x})-f(x))+\varepsilon s(1-s)\|x-\bar{x}\|
$$

we deduce similarly the relations

$$
\left\langle x^{*}, \bar{x}-x\right\rangle \leq f(\bar{x})-f(x)+\varepsilon\|x-\bar{x}\| .
$$

The proof of assertion (b) is similar.

The preceding result shows that approximately convex functions enjoy a very desirable property called firm softness in [39]. It is related to the concept of limiting subdifferential; recall that the limiting subdifferential $\partial^{L} f(\bar{x})$ of a function $f$ at $\bar{x}$ is the set of weak* cluster points of bounded sequences $\left(x_{n}^{*}\right)$ such that $x_{n}^{*} \in \partial^{F} f\left(x_{n}\right)$ for a sequence $\left(x_{n}\right) \rightarrow \bar{x}$ satisfying $\left(f\left(x_{n}\right)\right) \rightarrow f(\bar{x})$ (and then we write $\left.\left(x_{n}\right) \stackrel{f}{\rightarrow} \bar{x}\right)$.

Lemma 2.2 Let $f$ be l.s.c. and approximately convex at $\bar{x}$. Then $f$ is firmly soft at $\bar{x}$ in the following sense: for any sequence $\left(x_{n}\right) \stackrel{f}{\rightarrow} \bar{x}$ and any weak ${ }^{*}$ cluster point $\bar{x}^{*}$ of a bounded sequence $\left(x_{n}^{*}\right)$ such that $x_{n}^{*} \in \partial^{F} f\left(x_{n}\right)$ for every $n \in \mathbb{N}$, one has $\bar{x}^{*} \in \partial^{F} f(\bar{x})$. Equivalently, $\partial^{L} f(\bar{x})=$ $\partial^{F} f(\bar{x})$.

Proof. Given sequences $\left(x_{n}\right) \stackrel{f}{\rightarrow} \bar{x}, \bar{x}^{*},\left(x_{n}^{*}\right)$ as in the statement, for any $\varepsilon>0$ there exists some $\delta>0$ and $m \in \mathbb{N}$ such that, for every $x^{\prime} \in B(\bar{x}, \delta)$, and $n \geq m$ we have $x_{n} \in B(\bar{x}, \delta)$, and

$$
\forall x^{\prime} \in B(\bar{x}, \delta) \quad\left\langle x_{n}^{*}, x^{\prime}-x_{n}\right\rangle \leq f\left(x^{\prime}\right)-f\left(x_{n}\right)+\varepsilon\left\|x^{\prime}-x_{n}\right\| .
$$

Passing to the limit on a subnet, we get, since $\left(x_{n}^{*}\right)$ is bounded,

$$
\forall x^{\prime} \in B(\bar{x}, \delta) \quad\left\langle\bar{x}^{*}, x^{\prime}-\bar{x}\right\rangle \leq f\left(x^{\prime}\right)-f(\bar{x})+\varepsilon\left\|x^{\prime}-\bar{x}\right\| .
$$

Thus $\bar{x}^{*} \in \partial^{F} f(\bar{x})$.

A function $f$ is said to be a difference of approximately starshaped functions (in short a d.a.s. function) if there exist two approximately starshaped proper lower semicontinuous (l.s.c.) functions $g$ and $h$ defined on $E$ such that $f=g-h$. Since $g$ and $h$ may take the value $+\infty$ at the same time, we adopt here the rule $(+\infty)-(+\infty)=+\infty$. When $g$ and $h$ are convex, $f$ is called a d.c. function.

We will use a mild continuity condition introduced in [40]. We just recall its definition and we refer to [40] for its main properties. 
Definition 2.2 A multifunction $F: X \rightrightarrows Y$ between a topological space $X$ and a metric space $Y$ is said to be gap-continuous at $\bar{x} \in X$ if

$$
\operatorname{gap}(F(\bar{x}), F(x)) \rightarrow 0 \text { as } x \rightarrow \bar{x}
$$

where, for two subsets $A, B$ of $Y$ one sets

$$
\operatorname{gap}(A, B)=\inf \{d(a, b): a \in A, b \in B\},
$$

with the usual convention: $\operatorname{gap}(A, B)=\infty$ if $A=\varnothing$ or $B=\varnothing$.

This notion is weaker than lower and upper semicontinuity. It satisfies some stability properties under composition, sums, products, convex hull operations (see [40]).

\section{The Fréchet subdifferentials of d.c. functions.}

Let us start our study by recalling a simple but useful observation.

Proposition 3.1 ([38, (7)]) Let $g, h: E \rightarrow \mathbb{R}_{\infty}$ and let $f=g-h$ be finite at $\bar{x}$. Then

$$
\partial^{F} f(\bar{x}) \subset \partial^{F} g(\bar{x}) \boxminus \partial^{F} h(\bar{x}) .
$$

In particular, if $h$ is convex, one has $\partial^{F} f(\bar{x}) \subset \partial^{F} g(\bar{x}) \boxminus \partial h(\bar{x})$ and, if $g, h$ are convex, one has $\partial^{F} f(\bar{x}) \subset \partial g(\bar{x}) \boxminus \partial h(\bar{x})$.

Proof. When $h$ is finitely valued, the proof follows from the fact that $g=f+h$, so that

$$
\partial^{F} f(\bar{x})+\partial^{F} h(\bar{x}) \subset \partial^{F}(f+h)(\bar{x})=\partial^{F} g(\bar{x}),
$$

but we consider the general case (which could be deduced from the relations $f+h \leq g, f(\bar{x})+$ $h(\bar{x})=g(\bar{x}))$. Given $\bar{x}^{*} \in \partial^{F} f(\bar{x})$, let us show that for any $\bar{z}^{*} \in \partial^{F} h(\bar{x})$ one has $\bar{y}^{*}:=\bar{x}^{*}+\bar{z}^{*} \in$ $\partial^{F} g(\bar{x})$. By definition, for every $\varepsilon>0$ there exists $\delta>0$ such that for all $x \in B(\bar{x}, \delta)$

$$
\begin{aligned}
& \left\langle\bar{x}^{*}, x-\bar{x}\right\rangle \leq f(x)-f(\bar{x})+\frac{\varepsilon}{2}\|x-\bar{x}\|, \\
& \left\langle\bar{z}^{*}, x-\bar{x}\right\rangle \leq h(x)-h(\bar{x})+\frac{\varepsilon}{2}\|x-\bar{x}\| .
\end{aligned}
$$

The first of these relations shows that $f(x)>-\infty$ for all $x \in B(\bar{x}, \delta)$, so that $f(x)+h(x)=g(x)$. Adding sides by sides these two inequalities, we get, for all $x \in B(\bar{x}, \delta)$,

$$
\left\langle\bar{y}^{*}, x-\bar{x}\right\rangle \leq g(x)-g(\bar{x})+\varepsilon\|x-\bar{x}\|,
$$

what means that $\bar{y}^{*} \in \partial^{F} g(\bar{x})$.

The last assertion follows from the fact that for a convex function $k$ one has $\partial^{F} k(\bar{x})=\partial k(\bar{x})$.

The following immediate consequence can easily be established directly as in [38]. 
Corollary 3.1 If $\bar{x}$ is a local minimizer of $f=g-h$, then one has

$$
0 \in \partial^{F} g(\bar{x}) \boxminus \partial^{F} h(\bar{x}),
$$

or equivalently, $\partial^{F} h(\bar{x}) \subset \partial^{F} g(\bar{x})$.

The following example taken from [38] shows that in general the inclusion of Proposition 3.1 is strict.

Example 4. Let $E=\mathbb{R}$ and let $f, g, h$ be given by $f(0)=g(0)=h(0):=0$ and $f(x)=$ $|x| \sin ^{2}(1 / x), g(x)=|x|, h(x)=|x| \cos ^{2}(1 / x)$ for $x \in \mathbb{R} \backslash\{0\}$. Then $\partial^{F} g(0)=[-1,1], \partial^{F} h(0)=$ $\{0\}$, hence $\partial^{F} g(0) \boxminus \partial^{F} h(0)=[-1,1]$, while $\partial^{F} f(0)=\{0\}$.

It may happen in particular circumstances that inclusion (3.8) holds as an equality. This is the case for any function $f$ finite at $\bar{x}$ when one takes $g:=(r+1) f, h:=r f$ for some $r>0$ (because $\partial^{F} f(\bar{x})$ is convex and for any convex set $C$ one has $C=(r+1) C \boxminus r C$ ). The following result provides a sufficient condition offering more generality.

Theorem 3.1 Let $f=g-h$ be finite at $\bar{x}$, with $g$, $h$ approximately starshaped at $\bar{x}$. Suppose that $\partial^{F} h$ is gap-continuous at $\bar{x}$. Then

$$
\partial^{F} f(\bar{x})=\partial^{F} g(\bar{x}) \boxminus \partial^{F} h(\bar{x}) .
$$

Proof. Taking Proposition 3.1 into account, it suffices to prove that for every $\bar{x}^{*} \in \partial^{F} g(\bar{x}) \boxminus$ $\partial^{F} h(\bar{x})$ one has $\bar{x}^{*} \in \partial^{F} f(\bar{x})$. By definition of gap continuity and Lemma 2.1, given $\varepsilon>0$ we can find $\delta>0$ such that

$$
\begin{aligned}
& \forall x \in B(\bar{x}, \delta) \quad \partial^{F} h(x) \bigcap\left(\partial^{F} h(\bar{x})+\varepsilon B^{*}\right) \neq \varnothing, \\
& \forall x \in B(\bar{x}, \delta), \forall \bar{y}^{*} \in \partial^{F} g(\bar{x}) \quad\left\langle\bar{y}^{*}, x-\bar{x}\right\rangle \leq g(x)-g(\bar{x})+\varepsilon\|x-\bar{x}\|, \\
& \forall x \in B(\bar{x}, \delta), \forall z^{*} \in \partial^{F} h(x) \quad\left\langle z^{*}, \bar{x}-x\right\rangle \leq h(\bar{x})-h(x)+\varepsilon\|x-\bar{x}\| .
\end{aligned}
$$

Given $x \in B(\bar{x}, \delta)$ let us pick $z^{*} \in \partial^{F} h(x)$ and $\bar{z}^{*} \in \partial^{F} h(\bar{x})$ such that $\left\|z^{*}-\bar{z}^{*}\right\|<\varepsilon$. Then $h(x)$ is finite and $\bar{y}^{*}:=\bar{x}^{*}+\bar{z}^{*} \in \partial^{F} g(\bar{x})$. Adding sides by sides inequalities (3.11) and (3.12), we get

$$
\begin{aligned}
& \left\langle\bar{x}^{*}, x-\bar{x}\right\rangle-\left\langle z^{*}-\bar{z}^{*}, x-\bar{x}\right\rangle \leq f(x)-f(\bar{x})+2 \varepsilon\|x-\bar{x}\|, \\
& \left\langle\bar{x}^{*}, x-\bar{x}\right\rangle \leq f(x)-f(\bar{x})+3 \varepsilon\|x-\bar{x}\| .
\end{aligned}
$$

Since this last relation is satisfied for every $x \in B(\bar{x}, \delta)$, we have $\bar{x}^{*} \in \partial^{F} f(\bar{x})$.

The special case of d.c. functions follows immediately.

Corollary 3.2 If $f:=g-h$, with $g$ and $h$ convex and if $\partial h$ is gap-continuous at $\bar{x} \in \operatorname{dom} f$, then

$$
\partial^{F} f(\bar{x})=\partial g(\bar{x}) \boxminus \partial h(\bar{x}) .
$$

It is of interest to compare the Fréchet subdifferential $\partial^{F} f(\bar{x})$ of $f$ at $\bar{x}$ with the limiting subdifferential $\partial^{L} f(\bar{x})$ of $f$ at $\bar{x}$ when $f$ admits a decomposition $g-h$. 
Theorem 3.2 Let $f=g-h$, with $g$, $h$ finite at $\bar{x}, g$ firmly soft at $\bar{x}$ and $h$ continuous at $\bar{x}$. Suppose that relation (3.9) holds and that $\partial^{F} h$ is lower semicontinuous at $\bar{x}$ from $X$ to $X^{*}$ endowed with the weak* topology. Then $f$ is firmly soft at $\bar{x}$ in the sense that $\partial^{L} f(\bar{x})=\partial^{F} f(\bar{x})$.

Proof. Let $\bar{x}^{*} \in \partial^{L} f(\bar{x})$ : there exist sequences $\left(x_{n}\right) \stackrel{f}{\rightarrow} \bar{x},\left(x_{n}^{*}\right)$ bounded in $X^{*}$, having $\bar{x}^{*}$ as a weak* cluster point and satisfying $x_{n}^{*} \in \partial^{F} f\left(x_{n}\right)$ for each $n \in \mathbb{N}$. By relation (3.9), it suffices to show that for any $\bar{z}^{*} \in \partial^{F} h(\bar{x})$ we have $\bar{x}^{*}+\bar{z}^{*} \in \partial^{F} g(\bar{x})$. Let $\left(x_{n(j)}\right)_{j \in J}$ be a subnet of $\left(x_{n}\right)_{n \in N}$ and let $\left(z_{j}^{*}\right)_{j \in J}$ be a net weak* converging to $\bar{z}^{*}$ such that $z_{j}^{*} \in \partial h\left(x_{n(j)}\right)$ for all $j \in J$. Now Proposition 3.1 ensures that $x_{n}^{*}+\partial^{F} h\left(x_{n}\right) \subset \partial^{F} g\left(x_{n}\right)$ for each $n \in \mathbb{N}$. Thus $x_{n(j)}^{*}+z_{n(j)}^{*} \in \partial^{F} g\left(x_{n(j)}\right)$ for all $j \in J$; passing to the limit, we get $\bar{x}^{*}+\bar{z}^{*} \in \partial^{F} g(\bar{x})$ as $g$ is firmly soft at $\bar{x}$ and $\left(g\left(x_{n}\right)\right) \rightarrow g(\bar{x})$ by the continuity of $h$ at $\bar{x}$.

Remark. Instead of assuming that (3.9) holds and that $g$ is firmly soft at $\bar{x}$ one may suppose

$$
\partial^{F} f(\bar{x})=\partial^{L} g(\bar{x}) \boxminus \partial^{F} h(\bar{x}) .
$$

The weak* lower semicontinuity assumption on $\partial h$ is restrictive. However it is satisfied in some cases, as shown by the following corollary.

Corollary 3.3 Let $f=g-h$, with $g$, $h$ finite at $\bar{x}, g$ firmly soft at $\bar{x}$. Suppose relation (3.9) holds. Then, under each of the following assumptions, $f$ is firmly soft at $\bar{x}$ :

(a) $h$ is of class $C^{1}$ at $\bar{x}$ (i.e. $h$ is differentiable around $\bar{x}$ and its derivative is continuous at $\bar{x})$;

(b) $h$ is convex and Fréchet differentiable at $\bar{x}$;

(c) $h$ is the infimum of a finite family $\left(h_{i}\right)_{i \in I}$ of functions of class $C^{1}$ at $\bar{x}$ and $\partial^{F} h(x)$ is nonempty for $x$ in a neighborhood of $\bar{x}$.

Proof. In cases (a) or (b) $\partial^{F} h$ is strongly upper semicontinuous at $\bar{x}$ (see [42, Lemma 2.6]) and $\partial^{F} h(\bar{x})=\{D h(\bar{x})\}$. Moreover, since in case (b) $h$ is continuous around $\bar{x}$, in both cases there exists $r>0$ such that for any net $\left(x_{n}\right)_{n \in N} \rightarrow \bar{x}$, one can find $n_{0} \in N$ such that $\partial^{F} h\left(x_{n}\right)$ is nonempty and contained in $r B^{*}$ for $n \geq n_{0}$. Then, by weak* compactness of $r B^{*}$, one can find a subnet $\left(x_{n(j)}\right)_{j \in J}$ of $\left(x_{n}\right)_{n \in N}$ and a weak* converging net $\left(x_{j}^{*}\right)_{j \in J}$ such that $x_{j}^{*} \in \partial h\left(x_{n(j)}\right)$ for all $j \in J$. The limit $\bar{x}^{*}$ of $\left(x_{j}^{*}\right)_{j \in J}$ cannot be anything else that $D h(\bar{x})$.Thus, the lower semicontinuity assumption we used in the preceding theorem is fulfilled. In case (c), setting, for $x \in X, I(x):=\left\{i \in I: h_{i}(\bar{x})=h(\bar{x})\right\}$, we see that $I(x) \subset I(\bar{x})$ for $x$ close to $\bar{x}$ and that $h_{i}^{\prime}(x)=h_{j}^{\prime}(x)$ for $i, j \in I(x)$ for $x$ close to $\bar{x}$, $\partial^{F} h(x)$ being $\left\{h_{i}^{\prime}(x)\right\}$ for $i \in I(x)$. These observations prove that $\partial^{F} h$ is lower semicontinuous at $\bar{x}$; in fact $h$ is of class $C^{1}$ at $\bar{x}$.

Remark. In (a) it would suffice to assume that $h$ is of class $T^{1}$ at $\bar{x}$ in the sense that $h$ is Gâteaux (or Hadamard) differentiable around $\bar{x}$ and $(x, v) \mapsto h^{\prime}(x)(v)$ is continuous at $(\bar{x}, \bar{v})$ for any $\bar{v} \in X$ (see [37]).

\section{Equi-subdifferentiability}

The following definition delineates a uniformity property which is automatically satisfied when the subdifferential $\partial^{F} f(\bar{x})$ is a singleton, in particular when $f$ is differentiable at $\bar{x}$. Thus, it takes into account the multivaluedness of the Fréchet subdifferential. 
Definition 4.1 Let us say that a subset $F$ of $\partial^{F} f(\bar{x})$ is a subset of equi-subdifferentiability of $f$ at $\bar{x}$ if

$$
\forall \varepsilon>0 \exists \delta>0 \quad \forall x^{*} \in F \quad \forall x \in B(\bar{x}, \delta) \quad f(x)-f(\bar{x})-\left\langle x^{*}, x-\bar{x}\right\rangle \geq-\varepsilon\|x-\bar{x}\| .
$$

We say that $f$ is equi-subdifferentiable at $\bar{x}$ if $\partial^{F} f(\bar{x})$ is nonempty and is a subset of equisubdifferentiability of $f$ at $\bar{x}$.

Obviously, the convex hull of any finite subset of $\partial^{F} f(\bar{x})$ is a subset of equi-subdifferentiability of $f$ at $\bar{x}$. If $f$ is convex, or just approximately starshaped at $\bar{x}$, with $\partial f(\bar{x})$ nonempty, then $f$ is equi-subdifferentiable at $\bar{x}$, as shown by Lemma 2.1 (a). The same is true if $f=g-h$ with $g$ convex and $h$ Fréchet differentiable at $\bar{x}$. In fact, this last assertion is a special case of the next proposition. If $g=f+h$ and if $F$ (resp. $H$ ) is a subset of equi-subdifferentiability of $f$ (resp. $h$ ) at $\bar{x}$, then $G:=F+H$ is a subset of equi-subdifferentiability of $g$ at $\bar{x}$, as easily seen. If $f:=\sup _{i \in I} f_{i}$ is the supremum of a family of functions and if, for a finite subset $J$ of $I(\bar{x}):=\left\{i \in I: f_{i}(\bar{x})=f(\bar{x})\right\}, F_{j}$ is a subset of equi-subdifferentiability of $f_{j}$ at $\bar{x}$, then the convex hull of the union of the $F_{j}$ 's is a subset of equi-subdifferentiability of $f$ at $\bar{x}$. If $f=g \circ \theta$, with $\theta: X \rightarrow Y$ Fréchet differentiable at $\bar{x}$ and if $G$ is a subset of equi-subdifferentiability of $g$ at $\theta(\bar{x})$ then $F:=\theta^{\prime}(\bar{x})^{\top}(G)$ is a subset of equi-subdifferentiability of $f$ at $\bar{x}$.

Proposition 4.1 Let $f=g-h$ with $g$, $h$ finite at $\bar{x}$ and $h$ approximately starshaped at $\bar{x}$. If $\partial^{F} h$ is gap-continuous at $\bar{x}$ and if $G$ is a subset of equi-subdifferentiability of $g$ at $\bar{x}$, then $G \boxminus \partial^{F} h(\bar{x})$ is a subset of equi-subdifferentiability of $f$ at $\bar{x}$. In particular

$$
G \boxminus \partial^{F} h(\bar{x}) \subset \partial^{F} f(\bar{x}) .
$$

Proof. The proof is similar to the proof of Theorem 3.1. It suffices to replace $\partial^{F} g(\bar{x})$ by $G$ in relation (3.11).

Taking $G:=\partial^{F} g(\bar{x})$ and using Proposition 4.1, we get the following corollary.

Corollary 4.1 If $f=g-h$ with $g$, $h$ finite at $\bar{x}, g$ equi-subdifferentiable at $\bar{x}, h$ approximately starshaped at $\bar{x}$ and if $\partial^{F} h$ is gap-continuous at $\bar{x}$, then $f$ is equi-subdifferentiable at $\bar{x}$ and

$$
\partial^{F} f(\bar{x})=\partial^{F} g(\bar{x}) \boxminus \partial^{F} h(\bar{x}) .
$$

Corollary 4.2 If $f=g-h$ with $g, h$ finite at $\bar{x}$ and approximately starshaped at $\bar{x}$ and if $\partial^{F} h$ is gap-continuous at $\bar{x}$, then $f$ is equi-subdifferentiable at $\bar{x}$ and the preceding relation holds.

A converse of the preceding proposition can be given. Its proof follows from an inspection of the proof of Proposition 3.1.

Proposition 4.2 Let $f=g-h$ with $g$, $h$ finite at $\bar{x}$. If $F$ (resp. $H$ ) is a subset of equisubdifferentiability of $f$ (resp. $h$ ) at $\bar{x}$, then $G:=F+H$ is a subset of equi-subdifferentiability of $g$ at $\bar{x}$.

Corollary 4.3 Let $f=g-h$ with $g, h$ finite at $\bar{x}, h$ approximately convex at $\bar{x}$, $\partial^{F} h$ being gap-continuous at $\bar{x}$. Then $F$ is a subset of equi-subdifferentiability of $f$ at $\bar{x}$, if, and only if, $G:=F+\partial^{F} h(\bar{x})$ is a subset of equi-subdifferentiability of $g$ at $\bar{x}$.

Proof. The only if assertion is contained in the preceding proposition. The if assertion stems from Proposition 4.1 and the fact that if $G:=F+\partial^{F} h(\bar{x})$ then $F \subset G \boxminus \partial^{F} h(\bar{x})$. 


\section{Approximate Fréchet subdifferentials of d.c. functions.}

The next theorem exhibits a formula for the $\varepsilon$-Fréchet subdifferential for $f:=g-h$ similar to the one in Theorem 3.1. We first note that for every $\varepsilon \geq 0$ the inclusion

$$
\partial_{\varepsilon}^{F} f(\bar{x}) \subset \bigcap_{\mu \geq 0} \partial_{\varepsilon+\mu}^{F} g(\bar{x}) \boxminus \partial_{\mu}^{F} h(\bar{x})
$$

holds, since we have $f+h \leq g, f(\bar{x})+h(\bar{x})=g(\bar{x})$, hence, for any $\mu \geq 0$, by formula (2.4),

$$
\partial_{\varepsilon}^{F} f(\bar{x})+\partial_{\mu}^{F} h(\bar{x}) \subset \partial_{\varepsilon+\mu}^{F} g(\bar{x}) .
$$

In the next theorem we give a sufficient condition ensuring that relation (5.13) is an equality.

Theorem 5.1 Let $f:=g-h$, with $g, h$ approximately starshaped at $\bar{x}$. Suppose that, for some $\lambda>0$, the multimap $\partial_{\lambda}^{F} h$ is gap-continuous at $\bar{x}$. Then, for all $\varepsilon \geq 0$,

$$
\partial_{\varepsilon}^{F} f(\bar{x})=\partial_{\varepsilon+\lambda}^{F} g(\bar{x}) \boxminus \partial_{\lambda}^{F} h(\bar{x})=\partial_{\varepsilon+\lambda}^{F} g(\bar{x}) \boxminus \partial_{\lambda}^{F} h(\bar{x})=\bigcap_{\mu \geq 0} \partial_{\varepsilon+\mu}^{F} g(\bar{x}) \boxminus \partial_{\mu}^{F} h(\bar{x}) .
$$

Proof. Let $\varepsilon \geq 0, \lambda>0, \bar{x}^{*} \in \bigcap_{\lambda>0} \partial_{\varepsilon+\lambda}^{F} g(\bar{x}) \boxminus \partial_{\lambda}^{F} h(\bar{x})$ be given. In view of inclusion (5.13) it suffices to prove that $\bar{x}^{*} \in \partial_{\varepsilon}^{F} f(\bar{x})$. Let us define the functions $g_{\varepsilon+\lambda}$ and $h_{\lambda}$ by

$$
g_{\varepsilon+\lambda}(x)=g(x)+(\varepsilon+\lambda)\|x-\bar{x}\|, \quad h_{\lambda}(x)=h(x)+\lambda\|x-\bar{x}\| .
$$

Then $g_{\varepsilon+\lambda}$ and $h_{\lambda}$ are approximately starshaped at $\bar{x}$ and for all $x \in E$ one has

$$
g_{\varepsilon+\lambda}(x)-h_{\lambda}(x)=f_{\varepsilon}(x):=f(x)+\varepsilon\|x-\bar{x}\| .
$$

Theorem 3.1 ensures that $\bar{x}^{*} \in \partial^{F} g_{\varepsilon+\lambda}(\bar{x}) \boxminus \partial^{F} h_{\lambda}(\bar{x})=\partial^{F} f_{\varepsilon}(\bar{x})=\partial_{\varepsilon}^{F} f(\bar{x})$.

Remark. In the preceding proof we have used the assumption that the multifunction $\partial_{\lambda}^{F} h$ is gap-continuous at $\bar{x}$. While for $h$ convex the Fenchel $\lambda$-subdifferential $\partial_{\lambda} h$ is continuous on dom $h$ ([7]), the Fréchet $\lambda$-subdifferential $\partial_{\lambda}^{F} h$ does not seem to be much more regular than $\partial h$. However, in view of [?, Lemma 3.1, Prop. 3.1 (b)], and of the inclusion $\partial^{F} h(x)+\lambda B^{*} \subset \partial_{\lambda}^{F} h(x), \partial_{\lambda}^{F} h$ is gap-continuous at $\bar{x}$ whenever $\partial^{F} h$ is gap-continuous at $\bar{x}$, as easily seen with the help of relation (2.3).

Corollary 5.1 Let $f=g-h$ with $g, h$ convex. Suppose $\partial h$ gap-continuous at $\bar{x}$. Then, for all $\varepsilon \geq 0$,

$$
\partial_{\varepsilon}^{F} f(\bar{x})=\left(\partial g(\bar{x})+\varepsilon B^{*}\right) \boxminus \partial h(\bar{x}) .
$$




\section{Application to optimality conditions.}

Let us devote this final section to applications of the preceding results to optimality conditions. Our results differ from the ones in [38] by several assumptions. Following a number of papers, we will say that a point $\bar{x} \in E$ is a local sharp minimizer (or local firm minimizer) of a function $f$ if $f$ is finite at $\bar{x}$ and if there exist $\delta, \sigma>0$ such that $f(x) \geq f(\bar{x})+\sigma\|x-\bar{x}\|$ for every $x \in B(\bar{x}, \delta)$. Such a property is stronger than mere local strict minimization as it involves a conditioning (or well-posedness) property. On the contrary, the next definition points out weakenings of the notion of minimizer.

Definition 6.1 Let $\varepsilon$ be a positive real number. A point $\bar{x}$ is said to be an $\varepsilon$-minimizer of $f$ on a subset $S$ of $E$ if $\bar{x} \in S$ and if

$$
f(\bar{x})-\varepsilon \leq f(x) \text { for all } x \in S .
$$

We say that $\bar{x}$ is a blunt minimizer (or, more precisely an $\varepsilon$-blunt minimizer) of $f$ on a subset $S$ of $E$ if $\bar{x} \in S$ and

$$
f(\bar{x})-\varepsilon\|x-\bar{x}\| \leq f(x) \text { for all } x \in S .
$$

For $S=E$ we just say that $\bar{x}$ is a blunt minimizer.

We say that $\bar{x}$ is a local blunt minimizer (or, a local $\varepsilon$-blunt minimizer) of $f$ (on $S$ ) if it is an $\varepsilon$-blunt minimizer of $f$ on some neighborhood of $\bar{x}$ (in $S$ ).

An application of Ekeland's variational principle [8] entails part (a) of the following proposition; part (b) is obtained by taking $\delta=1$.

Proposition 6.1 Let $C$ be a nonempty closed subset of $E$ and let $f: E \rightarrow \mathbb{R}_{\infty}$ be a l.s.c. function .

(a) If $x_{0}$ is an E-minimizer of $f$ on $C$ then, for all $\delta>0$, there exists $\bar{x} \in C \cap \bar{B}\left(x_{0}, \delta\right)$ such that $\bar{x}$ is an $\frac{\varepsilon}{\delta}$-blunt minimizer of $f$ on $C$. C.

(b) If $f$ is bounded below on $C$ then, for all $\varepsilon>0$, there exists an $\varepsilon$-blunt minimizer of $f$ on

Proposition 6.2 Let $f: E \rightarrow \mathbb{R}_{\infty}$ be an arbitrary function finite at some $\bar{x} \in E$. Then $\bar{x}$ is a local sharp minimizer of $f$ if, and only if, there exists some $\rho>0$ such that $\rho B^{*}$ is a subset of equi-subdifferentiability of $f$ at $\bar{x}$.

Proof. Suppose $\bar{x}$ is a local sharp minimizer of $f$ : there exist $\delta>0$ and $\sigma>0$ such that $f(x) \geq f(\bar{x})+\sigma\|x-\bar{x}\|$ for every $x \in B(\bar{x}, \delta)$. Then, for all $\rho \in(0, \sigma]$, all $x^{*} \in B^{*}$ and all $\varepsilon>0$, $x \in B(\bar{x}, \delta)$ we have $f(x)-f(\bar{x})-\left\langle x^{*}, x-\bar{x}\right\rangle+\sigma\|x-\bar{x}\| \geq-\varepsilon\|x-\bar{x}\|$, so that $\rho B^{*}$ is a subset of equi-subdifferentiability of $f$ at $\bar{x}$.

Conversely, suppose $\rho B^{*}$ is a subset of equi-subdifferentiability of $f$ at $\bar{x}$ for some $\rho>0$. Given $\sigma \in(0, \rho)$, let $\varepsilon>0$ be such that $\sigma+\varepsilon \leq \rho$. Let $\delta>0$ be such that

$$
\forall x^{*} \in \rho B^{*} \forall x \in B(\bar{x}, \delta) \quad\left\langle x^{*}, x-\bar{x}\right\rangle-\varepsilon\|x-\bar{x}\| \leq f(x)-f(\bar{x}) .
$$

Then, taking the supremum over $x^{*} \in \rho B^{*}$, we get 


$$
\forall x \in B(\bar{x}, \delta) \quad \sigma\|x-\bar{x}\| \leq(\rho-\varepsilon)\|x-\bar{x}\| \leq f(x)-f(\bar{x})
$$

and $\bar{x}$ is a local sharp minimizer of $f$.

Corollary 6.1 Let $f=g-h$ with $g, h$ approximately convex at $\bar{x}$. If $\partial^{F} h$ is gap-continuous at $\bar{x}$ and if

$$
0 \in \operatorname{int}\left(\partial^{F} f(\bar{x})\right)
$$

then $\bar{x}$ is a local sharp minimizer of $f$.

Proof. By assumption, there is some $\rho>0$ such that $\rho B^{*} \subset \partial^{F} f(\bar{x})$. Since $\partial^{F} h$ is gap-continuous, $\rho B^{*}$ is a subset of equi-subdifferentiability of $f$ at $\bar{x}$ by Corollary 4.2 and the preceding proposition completes the proof.

Remark. The same conclusion holds when $g$ is equi-subdifferentiable at $\bar{x}$ instead of being approximately convex. Note that assumption (6.14) can also be written: there is some $\rho>0$ such that $\partial^{F} h(\bar{x})+\rho B^{*} \subset \partial^{F} g(\bar{x})$.

We easily recover a result of [38].

Corollary 6.2 Let $E=\mathbb{R}^{n}, f=g-h$ where $g, h$ are convex and $h$ is continuous at $\bar{x}$. If

$$
0 \in \operatorname{int}\left(\partial^{F} f(\bar{x})\right)
$$

then $\bar{x}$ is a local sharp minimizer of $f$.

Proof. This is a direct consequence of the fact that $\partial h$ is gap continuous at $\bar{x}$ when $E$ is finite dimensional and $h$ is continuous at $\bar{x}$ by [40] and of Corollary 6.1 .

The following result gives necessary and sufficient conditions for local blunt optimality for d.c. or d.a.s. functions.

Theorem 6.1 Suppose $f:=g-h$ with $g, h$ approximately starshaped and finite at $\bar{x}$ and that $\partial^{F} h$ is gap-continuous at $\bar{x}$. Then the following assertions are equivalent:

i) $\partial^{F} h(\bar{x}) \subset \partial^{F} g(\bar{x})$;

ii) $0 \in \partial^{F} f(\bar{x})$;

iii) for all $\varepsilon>0, \bar{x}$ is a local $\varepsilon$-blunt minimizer of $f$;

iv) for some $\lambda>0, \partial_{\lambda}^{F} h(\bar{x}) \subset \partial_{\lambda}^{F} g(\bar{x})$;

v) for all $\lambda>0, \partial_{\lambda}^{F} h(\bar{x}) \subset \partial_{\lambda}^{F} g(\bar{x})$.

Proof. The equivalence ii) $\Leftrightarrow$ iii) is immediate and valid for any function $f$. The equivalence i) $\Leftrightarrow$ ii) is a consequence of Theorem 3.1. The equivalence ii) $\Leftrightarrow$ iv) stems from Theorem 5.1 with $\varepsilon=0$ since, as observed in the remark following Theorem 5.1, $\partial_{\lambda}^{F} h$ is gap-continuous whenever $\partial^{F} h$ is gap-continuous. The equivalence ii) $\Leftrightarrow \mathrm{v}$ ) is also a consequence of Theorem 5.1

Example 5. For the example considered in the introduction, $f=g-h$ with $g(x)=|x|$ and $h(x)=\frac{1}{2} x^{2}, 0$ is a local $\varepsilon$-blunt minimizer of $f$ for all $\varepsilon>0$ (in fact it is a local minimizer) and we also have for all $\lambda>0 \partial_{\lambda}^{F} h(0) \subset \partial_{\lambda}^{F} g(0)$ since a direct calculation shows that

$$
\partial_{\lambda}^{F} g(0)=[-1-\lambda, 1+\lambda] \text { and } \partial_{\lambda}^{F} h(0)=[-\lambda, \lambda] .
$$


For $f=g-h$ with $g=0, h(x)=\frac{1}{2} x^{2}, 0$ is a local $\varepsilon$-blunt minimizer of $f$ for all $\varepsilon>0$ as condition i) above is satisfied, but 0 is not a local minimizer of $f$.

Now let us consider a general constrained minimization problem of the form:

$$
\left(\mathcal{P}_{C}\right) \quad \text { minimize } \quad f(x):=g(x)-h(x) \text { subject to } \quad x \in C
$$

where $g, h: E \rightarrow \mathbb{R}$ are specified below and $C$ is a nonempty closed convex subset of $E$.

Proposition 6.3 Assume $f:=g-h$ with $g$ approximately convex and continuous at $\bar{x}$. Then a necessary condition for $\bar{x} \in C$ to be a local $\varepsilon$-blunt minimizer of $f$ on $C$ for all $\varepsilon>0$ is that

$$
\partial^{F} h(\bar{x}) \subset \partial^{F} g(\bar{x})+N_{C}(\bar{x})
$$

where $N_{C}(\bar{x})$ is the normal cone of convex analysis. Moreover, if $h$ is approximately starshaped at $\bar{x}$ and $\partial^{F} h$ is gap-continuous at $\bar{x}$, then the above inclusion is a sufficient condition for $\bar{x}$ to be a local $\varepsilon$-blunt minimizer of $f$ on $C$ for all $\varepsilon>0$.

Proof. If for all $\varepsilon>0, \bar{x} \in C$ is a local $\varepsilon$-blunt minimizer of $f$ on $C$, then, for all $\varepsilon>0, \bar{x}$ is a local $\varepsilon$-blunt minimizer of $f+\iota_{C}$ on $E$ where $\iota_{C}$ is the indicator function of $C$. Therefore $0 \in \partial^{F}\left(f+\iota_{C}\right)(\bar{x})$. Applying Proposition 3.1, one gets $\partial^{F} h(\bar{x}) \subset \partial^{F}\left(g+\iota_{C}\right)(\bar{x})=\partial^{F} g(\bar{x})+N_{C}(\bar{x})$, by the usual sum rule for limiting subdifferentials $([10])$ since $g$ is locally Lipschitzian around $\bar{x}$ by [32] and since $g$ is firmly soft at $\bar{x}$ by Lemma 2.2 .

Conversely, if $\partial^{F} h$ is gap-continuous and if the relation $\partial^{F} h(\bar{x}) \subset \partial^{F} g(\bar{x})+N_{C}(\bar{x})$ is satisfied, Theorem 3.1 ensures that $0 \in \partial^{F}\left(f+\iota_{C}\right)(\bar{x})$ since $f+\iota_{C}=\left(g+\iota_{C}\right)-h$ and $\partial^{F}\left(g+\iota_{C}\right)(\bar{x})=$ $\partial^{F} g(\bar{x})+N_{C}(\bar{x})$. By Theorem $6.1 \bar{x}$ is an $\varepsilon$-blunt minimizer of $f+\iota_{C}$ and then $\bar{x}$ is an $\varepsilon$-blunt minimizer of $f$ on $C$.

Now we are interested in the following maximization problems:

$$
\left(\mathcal{M}_{C}\right) \quad \text { maximize } h(x) \text { subject to } \quad x \in C
$$

where $h: E \rightarrow \mathbb{R}$ is a function and $C$ is a nonempty closed convex subset of $E$.

It is clear that $\sup _{x \in C} h(x)=-\inf _{x \in E}\left\{\iota_{C}(x)-h(x)\right\}$. So, a necessary condition for $\bar{x}$ to be an $\varepsilon$-blunt maximizer of $h$ on $C$ (i.e. $\bar{x}$ is an $\varepsilon$-blunt minimizer of $-h$ on $C$ ) is

$$
\partial^{F} h(\bar{x}) \subset N_{C}(\bar{x})
$$

Moreover if $h$ is approximately starshaped at $\bar{x}$ and $\partial^{F} h$ is gap-continuous at $\bar{x}$, then the above inclusion is a sufficient condition for $\bar{x}$ to be an $\varepsilon$-blunt maximizer of $h-\iota_{C}$, i.e. an $\varepsilon$-blunt maximizer of $h$ on $C$.

Acknowledgements. The authors are most grateful to C. Zălinescu for his helpful remarks on an earlier version of the manuscript. 


\section{References}

[1] An L. T. H. and Pham D. T., Convex analysis approach to d. c. programming: Theory, algorithms and applications, Acta Math. Vietnam. 22, No.1 (1997), 289-355.

[2] Asplund, E., Differentiability of the metric projection in finite dimensional Euclidean space, Proc. Amer. Math. Soc. 38 (1973), 218-219.

[3] Aubin, J.-P., Mutational and morphological analysis. Tools for shape evolution and morphogenesis, Systems and Control: Foundations and Applications, Birkhäuser, Boston, MA (1999).

[4] Aussel, D., Daniilidis, A. and Thibault, L., Subsmooth sets: functional characterizations and related concepts, Trans. Amer. Math. Soc. 357, No.4 (2005), 1275-1301.

[5] Caprari, E. and Penot J.-P., Tangentially d.-s. functions, Optimization 56 (1) (2007), 25-38.

[6] Clarke, F. H., Optimization and nonsmooth analysis, Wiley, New York 1983.

[7] Contesse, L. and Penot, J.-P., Continuity of the approximate subdifferential, J. Math. Anal. Appl. 156, No.2 (1991), 329-332.

[8] Ekeland, I., On the variational principle, J. Math. Anal. Appl. 47 (1970), 324-353.

[9] Flores-Bazán, F. and Oettli, W., Simplified optimality conditions for minimizing the difference of vector-valued functions, J. Optimization Theory Appl. 108, No.3 (2001), 571-586.

[10] Fabian, M., Subdifferentials, local $\varepsilon$-supports and Asplund spaces, J. Lond. Math. Soc., II. Ser. 34, 568-576 (1986).

[11] Gautier, S., Affine and eclipsing multifunctions, Numer. Funct. Anal. Optimization 11, No.7/8 (1990), 679-699.

[12] Hartman, P., On functions representable as a difference of convex functions, Pacific J. Math 9 (1959), 707-713.

[13] Hiriart-Urruty, J.-B., Generalized differentiability, duality and optimization for problems dealing with differences of convex functions, in Convexity and duality in optimization, editor J. Ponstein, Lecture Notes in Econom. and Math. Systems 256 (1986), 37-70.

[14] Hiriart-Urruty, J.-B., From convex to nonconvex optimization, in Nonsmooth optimization and related topics, editors F. H. Clarke, V.F. Demyanov, F.Giannessi, Plenum Press, (1989), 219-239.

[15] Hiriart-Urruty, J.-B., A general formula on the conjugate of the difference of functions, Can. Math. Bull. 29 (1986), 482-485.

[16] Hiriart-Urruty, J.-B. and Ledyaev, Y. S., A note on the characterization of the global maxima of a (tangentially) convex function over a convex set, J. Convex Anal. 3, No.1 (1996), 55-61. 
[17] Horst, R. and Tuy, H., Global Optimization (Deterministic Approaches), Springer Verlag, Berlin (1993).

[18] Horst, R. and Pardalos, P. M., Handbook of Global Optimization, Nonconvex Optimization and Its Applications 2, Kluwer, Dordrecht (1995).

[19] Horst, R., Pardalos, P. M. and Thoai, N. V., Introduction to Global Optimization, Nonconvex Optimization and Its Applications 48, Kluwer, Dordrecht (2000).

[20] Jofré, A., Luc, D. T. and Théra, M., $\varepsilon$-Subdifferential and $\varepsilon$-Monotonicity, Nonlinear Analysis theory Methods and Applications 33 (1998), 71-89.

[21] Jourani, A., Subdifferentiability and subdifferential monotonicity of $\gamma$-paraconvex functions, Control Cybern. 25, No.4, 721-737 (1996). Jourani, Abderrahim

[22] Jourani, A., Open mapping theorem and inversion theorem for $\gamma$-paraconvex multivalued mappings and applications, Stud. Math. 117, No.2, 123-136 (1996).

[23] M. Laghdir, N. Benkenza and N. Nadjeh, Duality in dc-constrained programming via duality in reverse convex programming, J. Nonlinear Convex Anal. 5 (2) (204), 275-283.

[24] Lemaire B. and Volle, M., Duality in dc programming, in "Generalized Convexity, Generalized Monotonicity: Recent Results. Proceedings of the 5th Symposium, Luminy, France, June 1721, 1996”, J.-P. Crouzeix et al., (eds.), Kluwer, Dordrecht (1998), 331-345.

[25] Le Thi Hoai An and Tao P.D., The DC (Difference of convex functions) programming and DCA revisited with DC models of real world nonconvex optimization problems, Ann. Oper. Res. 133 (2005), 23-46.

[26] Martínez-Legaz, J. E. and Penot, J.-P., Regularization by erasement, Math. Scandinavica 98 (2006), 97-124.

[27] Martínez-Legaz, J. E. and Seeger, A., A formula on the approximate subdifferential of the difference of convex functions, Bull. Austral. Math. Soc. 45 (1992), 37-41.

[28] Martínez-Legaz, J. E. and Volle, M., Duality for d. c. optimization over compact sets, in Optimization Theory. Recent Developments from Mátraháza, Giannessi, F. et al. (eds.), Appl. Optim. 59, Kluwer, Dordrecht, 139-146 (2001).

[29] Martínez-Legaz, J.E. and Volle, M., Duality in D. C. programming: The case of several D. C. constraints, J. Math. Anal. Appl. 237, No.2 (1999) 29 (4) (1986), 482-485.

[30] Matheron, G., Random sets and integral geometry, Wiley Series in Probability and Mathematical Statistics, Wiley, New York (1975).

[31] Michelot, C., Caractérisation des minima locaux des fonctions de la classe d.c., preprint, Univ. of Bourgogne, Dijon (1987). 
[32] Ngai, H.V., Luc, D.T. and Théra, M., Approximate convex functions, J. Nonlinear and Convex Anal. 1 (2) (2000), 155-176.

[33] Ngai, H.V. and Penot, J.-P., Approximately convex functions and approximately monotone operators, Nonlinear Anal. 66 (2007), 547-564.

[34] Ngai, H.V. and Penot, J.-P., Semismoothness and directional subconvexity of functions, preprint, Univ. of Pau May 2003, revised May 2005.

[35] Penot, J.-P., Variations on the theme of nonsmooth analysis : another subdifferential, in "Nondifferential optimization : motivations and applications", Proceedings, Sopron, Hungary, 1984, V.F. Demyanov and D. Pallasche editors, Lecture Notes in Econ. and Math. Systems 255, 41-54, Springer Verlag, Berlin, 1985.

[36] Penot, J.-P., Duality for anticonvex programs, J. of Global Optim. 19 (2001), 163-182.

[37] Penot, J.-P., Favorable classes of mappings and multimappings in nonlinear analysis and optimization, J. Convex Analysis 3 (1) (1996), 97-116.

[38] Penot, J.-P., On the minimization of difference functions, J. Global Optimization 12 (1998), 373-382.

[39] Penot, J.-P., Softness, sleekness and regularity properties in nonsmooth analysis, Nonlinear Anal., to appear (2007).

[40] Penot J.-P., Gap continuity of multimaps, (submitted).

[41] Penot, J.-P. and Bougeard, M.L., Approximation and decomposition properties of some classes of locally d.c. functions, Math. Program., Ser. A 41, No.2, 195-227 (1988).

[42] Phelps, R., Convex Functions, Monotone Operators and Differentiability, Lecture Notes in Mathematics 1364, Springer Verlag, Berlin, Heidelberg, New York, 1989.

[43] Plazanet, Ph., Existence et calcul des points critiques de différences de fonctions convexes. Application à l'optimisation et à l'étude d'une e.d.p., thesis, Univ. P. Sabatier, Toulouse, 1990.

[44] Plazanet, Ph., Contributions à l'analyse des fonctions convexes et des différences de fonctions convexes. Application à l'étude d'une e.d.p., preprint, Univ. P. Sabatier, Toulouse, 1990.

[45] Pontrjagin, L.S., Linear differential games II, Soviet Maths. 8 (4) (1967)

[46] Pshenichnii, B.N. Leçons sur les jeux différentiels, in Contrôle optimal et jeux différentiels, Cahier de l'INRIA nº 4 (1971),145-226.

[47] Rockafellar, R.T., Favorable classes of Lipschitz continuous functions in subgradient optimization, in Nondifferentiable Optimization (1982), Nurminski E. (eds), Pergamon Press, New York. 
[48] Spingarn, J.E., Submonotone subdifferentials of Lipschitz functions, Trans. Amer. Math. Soc. 264 (1981), 77-89.

[49] Strekalovsky, A. S., Extremal problems on complements of convex sets, Kybernetika 1 (1993), 113-126.

[50] Strekalovsky, A. S., Global optimality conditions for nonconvex optimization, J. Glob. Optim. 12, No.4 (1998), 415-434.

[51] Strekalovsky, A.S., One way to construct a global search algorithm for d. c. minimization problems, in "Nonlinear Optimization and Related Topics. Workshop, Erice, Sicily, Italy, June 23-July 2, 1998", Di Pillo, G. et al. (eds.), Appl. Optim. 36, Kluwer, Dordrecht (2000), 429-443.

[52] Strekalovsky, A.S., Some remarks on d. c. programming, in "Optimization and Optimal Control. International Conference on Optimization and Optimal Control, Ulaanbaatar, Mongolia, August 13-17, 2002", Pardalos, P. M. et al. (eds.), World Scientific Series on Computers and Operations Research 1, River Edge, NJ (2003), 347-367.

[53] Toland, J.F., Duality in nonconvex optimization, J. Math. Anal. Appl. 66 (1978), 399-415.

[54] Toland, J F., On subdifferential calculus and duality in non-convex optimization, Bull. Soc. Math. Fr., Suppl., Mém. 60 (Proc. Colloq., Pau 1977) (1979), 177-183.

[55] Tuy, H., On some recent advances and applications of d. c. optimization, in: Nguyen, Van Hien (ed.) et al., Optimization. Proceedings of the 9th Belgian-French-German Conference, Namur, Belgium, September 7-11, 1998. Lect. Notes Econ. Math. Syst. 481, Springer, Berlin (2000), 473-497.

[56] Volle, M., Conjugaison par tranches et dualité de Toland, Optimization 18 (1987), 633-642.

[57] Zălinescu, C., Convex Analysis in General Vector Spaces, World Scientific, Singapore, (2002). 